\title{
Dinámica del retorno de los migrantes y reinserción laboral en el municipio de Taniche, Oaxaca
}

\author{
Teresa Griselda Lustre Díaz, Marco Antonio Espinosa Trujillo \\ y Elizabeth Ojeda Velasco
}

Universidad NovaUniversitas

\section{Resumen}

El objetivo del presente trabajo fue conocer la reinserción laboral y los factores que explican su proceso. El estudio se realizó en Taniche, Oaxaca, una comunidad rural con un índice de intensidad migratoria alta y con registros de migración de retorno. Es un estudio descriptivo de corte transversal, con metodología cualitativa. Se consideraron a los migrantes que retornaron a Taniche durante el periodo 2015 a 2017. Los resultados de esta investigación muestran que los migrantes retornados se reinsertan en empleos precarios reproduciendo así las mismas condiciones que motivaron su emigración.

Palabras clave: precariedad, empleo, migración, desarrollo, rural.

\section{Dynamics of Return Migration and labor reinsertion in the municipality of Taniche, Oaxaca}

\begin{abstract}
The main objective of this work was to know the labour reinsertion and factors that explain this process. The study was performed in Taniche Oaxaca, a rural community with a high migration intensity index and with return migration records. It's a descriptive and cross-sectional study, with qualitative methodology. Migrants who returned to Taniche during the period 2015 to 2017 were considered. The results of this research show that returned migrants are reinserted into precarious work, thus reproducing the same conditions that motivated their emigration.
\end{abstract}

Keywords: Precarious, work, migration, development, rural.

Artículo recibido el 14 de noviembre de 2019 y aprobado el 10 de enero de 2020. 


\section{INTRODUCCIÓN}

$\mathrm{E}$ l fenómeno de la migración México-Estados Unidos, es uno de los procesos demográficos más complejos y ha sido estudiado ampliamente. Sin embargo, recientemente se ha mostrado un creciente interés por la migración de retorno.

La migración de retorno no es un fenómeno novedoso, pues al igual que la emigración es considerada como un fenómeno histórico con más de cien años de antigüedad (Durand, 1994; Instituto de Estudios y Divulgación sobre Migración [INEDIM], 2014) caracterizado por gran número de historias de idas y regresos; no obstante, en la actualidad ha tomado relevancia a consecuencia de la ruptura de esa circularidad ${ }^{1}$ (Masferrer, Sánchez y Rodríguez, 2017).

Los factores estructurales y coyunturales han propiciado una desaceleración de la emigración mexicana hasta un escenario de migración neta cercana a cero (Zenteno, 2012; Alarcón, 2012), esto se refleja en el aumento de un flujo importante de migrantes de retorno, tanto por la vía del retorno forzado (resultado de la política migratoria), como el retorno producto de las condiciones económicas expresadas en diversas dificultades como el desempleo y los bajos salarios (Gandini, Lozano-Ascencio y Gaspar, 2015) producto de las crisis económicas y el reciente endurecimiento de las políticas antimigratorias estadounidenses.

La crisis económica, el aumento de control fronterizo, la criminalidad en la zona fronteriza y las políticas migratorias implementadas en los últimos años (Alarcón, 2012; Masferrer, Sánchez y Rodríguez, 2017) han generado al menos dos escenarios: 1) los migrantes mexicanos indocumentados en Estados Unidos toman la decisión de hacer más larga su estancia migratoria, postergando así el regreso a su comunidad de origen, como respuesta al aumento de los costos y riesgos que enfrentan al cruzar la frontera (Durand, 2011; García, 2012; Rivera, 2015; Vásquez, 2016); y 2) la migración de retorno. Esto ha propiciado un mayor estudio del retorno migratorio y con ello una nueva fase migratoria entre México y Estados Unidos (Durand, 2006).

1 Para Masferrer, Sánchez y Rodríguez (2017) la circularidad se refiere al proceso de entrada y salida entre el lugar destino y de origen de los migrantes. 
Calva y Alarcón (2018: 57) analizaron una encuesta aplicada a migrantes mexicanos que fueron deportados desde Estados Unidos. Sus resultados muestran dos escenarios a los que se enfrentan los migrantes: 1) regresar a "sus terruños" como consecuencia de las deportaciones y 2) quedarse en las zonas fronterizas, pues cerca de 15.5 por ciento manifestó la intención de volver a cruzar la frontera de forma indocumentada a más tardar en siete días.

Datos de la Unidad de Política Migratoria de la Secretaría de Gobernación de México (2018) muestran que los estados de Guerrero, Michoacán y Oaxaca son las principales entidades receptoras de mexicanos repatriados desde Estados Unidos. En 2010 se registraron 469.2 mil eventos de repatriación, de los cuales 10.7, 8.3 y 7.6 por ciento correspondieron a los estados de Michoacán, Oaxaca y Guerrero, respectivamente. Para 2017, de las 167 mil repatriaciones, 27.4 por ciento se trató de migrantes originarios de estas mismas entidades. Estos datos son relevantes, pero únicamente documentan el retorno involuntario de los migrantes; por otra parte, no existen estadísticas confiables que den cuenta del retorno voluntario.

La migración de retorno tiene, por ende, implicaciones económicas tanto en el lugar de origen presionando la estructura laboral (Salas, Montoya y Soberón, 2011: 136), como en el país de destino por representar una ventaja porque alivia los problemas en la demanda de trabajo cuando se presenta alguna crisis económica (Jaúregui y Recaño, 2014). Como señala Barbosa y Aguirre (2011: 98), el retorno no solo puede tener múltiples efectos económicos a través de la inversión de divisas en las comunidades de origen, sino también por medio de la aplicación de las habilidades y conocimientos adquiridos en el extranjero.

El estudio de la migración de retorno no solo parte de la agenda de investigación por sus implicaciones demográficas; también cobra importancia del lado del Estado por las políticas públicas como la migratoria, de asilo y sobre la ayuda al desarrollo; además por el tema de las relaciones internacionales entre países de origen, tránsito y de destino (Cassarino, 2008: 63).

Actualmente, gran parte de las investigaciones sobre la migración de retorno se centran en el estudio de la reinserción sociolaboral y las implicaciones que tiene en el lugar o país de origen del migrante. $\mathrm{Al}$ respecto, se han planteado preguntas como: ¿qué sucede cuando la persona ha retornado y se enfrenta a la experiencia de reinsertarse 
social y productivamente? ¿En qué condiciones se da ese proceso de reinserción? ¿Existe en el contexto de retorno posibilidad real de que el individuo utilice los conocimientos, las habilidades y el capital financiero que adquirió durante su experiencia migratoria? ¿De qué manera responden o podrían responder los gobiernos locales ante estas situaciones? (Lozano y Martínez, 2015).

El presente trabajo tiene como objetivo conocer y describir cuáles son los factores que intervienen en el proceso de reinserción laboral de los retornados en Taniche, Oaxaca; una comunidad rural que se caracteriza por un índice de intensidad migratoria alta; además de haber registrado en los últimos años migraciones de retorno. La hipótesis sugiere que los migrantes retornados no logran su reinserción laboral, por pertenecer a una zona rural donde la oferta de trabajo es limitada y el contexto es adverso, a pesar de contar con conocimientos y habilidades que adquirieron durante su estancia en el extranjero.

\section{MIGRACIÓN DE RETORNO Y REINSERCIÓN LABORAL}

El concepto de migración de retorno no posee claridad al momento de definirlo, pues como menciona Castillo (1997) se caracteriza por su vaguedad conceptual que se refleja en el uso de diferentes expresiones como sinónimos: reemigración, contracorriente, reflujo, regreso, readmisión, entre otras.

La primera aproximación de la "migración de retorno" remite a "movimientos en el espacio territorial de cierta población que regresa al punto de partida u origen" (Lozano y Martínez, 2015: 26). Se considera migrante de retorno aquel individuo que regresa de manera voluntaria o involuntaria a su comunidad de origen; esto sin considerar el tiempo de duración de la experiencia migratoria (Cobo, 2008: 163). A decir de Rivera (2011), estos planteamientos llevan a considerar al retorno como la conclusión del proceso migratorio, bajo ciertos supuestos: 1) se trata de un regreso a su localidad de origen, 2) se reinsertan en la dinámica local y 3 ) el regreso es observado a priori como un retorno permanente y definitivo, lo cual nos lleva a una percepción lineal del retorno migratorio, esto es, la culminación del proyecto migratorio.

Si se parte del hecho que la migración no es un fenómeno lineal, sino más bien circulatorio, no definido y multidireccional (Rivera, 2008); la de retorno resulta replicar este mismo entramado complejo, como lo mencionan Lozano y Martínez (2015: 13): 
La experiencia histórica de las movilidades humanas, y particularmente la manera en cómo opera el proceso migratorio contemporáneo, ha roto con esa percepción tradicional del retorno, sencillamente porque no siempre se "vuelve" al origen o porque, en especial, no necesariamente implica el fin del plan migratorio, sino una etapa más en el complejo entramado de la movilidad.

Este entramado complejo de la migración, cuya lógica escapa del fenómeno lineal, ha llevado a varios investigadores a estudiar y medir el retorno con la elaboración de distintas tipologías que permiten la operacionalización y el análisis de la migración de retorno. Durand (2004), por ejemplo, remite a cinco categorías: el retorno definitivo y voluntario después de una larga estancia con la idea de un proyecto de vida en el terruño; el de los trabajadores temporales, sujetos a programas específicos que los obligan a retornar; el transgeneracional que alude al retorno ya no del migrante, sino de los descendientes; el forzado que remite a razones políticas y/o raciales; y el voluntario por fracaso cuyas razones son principalmente por experiencias negativas en la aventura migratoria.

La Organización de las Naciones Unidas para la Educación, la Ciencia y la Cultura (2008), clasifica a la migración de retorno de una manera más simple: retorno voluntario e involuntario. El primero se refiere a aquellos migrantes que regresan a su país de origen, después de un tiempo de haber vivido en otro país, mientras que el retorno involuntario, se refiriere a los migrantes que regresan de forma obligada a su país de origen, como resultado de una política migratoria. Actualmente, tiene mayor incidencia la de retornados involuntarios, particularmente por la presencia de la política antinmigrante (Jacobo y Cárdenas, 2018: 6).

Las tipologías antiinmigrantes determinan los factores explicativos que condicionan la decisión de regresar. En el retorno voluntario se encuentran factores tales como: búsqueda de la reunificación familiar, alguna enfermedad, una situación económica desfavorable en el país de destino, la aplicación de políticas antiinmigrantes o cualquier otra situación distinta a la deportación (Jacobo y Cárdenas 2018: 7). Respecto al retorno involuntario, las razones se relacionan con el rechazo de una solicitud de asilo, por su permanecía irregular en el país de destino (más tiempo del permitido o por haber ingresado de manera subrepticia) o porque se comete algún delito (d'Anglejan, 2009: 26). 
En el retorno los migrantes reúnen información acerca de las circunstancias existentes en los países de origen y destino; y de la movilización de recursos tangibles (financieros, por ejemplo) e intangibles, como el capital humano y social, que contribuya a garantizar el reasentamiento en su tierra natal, por lo que los factores explicativos serán determinantes al momento de reinsertarse y reintegrarse laboralmente (Cassarino, 2008: 73).

Para Cassarino (2004: 269), la migración de retorno puede ser explicada teóricamente desde varias perspectivas como la economía neoclásica, la nueva economía de migración laboral, el estructuralismo, transnacionalismo y la teoría de redes sociales. Aunque desde la perspectiva de Castillo (1997: 39), la teoría del retorno debería integrar una teoría general de la migración, pues lo que existe son "un par de intentos de construcción teórica que tienen como objetivo el de ahondar en la comprensión del regreso de migrantes". Esto coincide con quienes sostienen que no existe una teoría general que explique el fenómeno de la migración, sino un conjunto de teorías aisladas y a veces segmentadas por los límites de cada disciplina (Massey et al., 1993; Durand, 2004).

La economía neoclásica explica que la migración es una decisión individual y es resultado de las diferencias salariales entre el país receptor y el país expulsor (Gómez, 2010: 92). El migrante es guiado por la racionalidad económica costo-beneficio, por eso busca maximizar su ingreso esperado en el país de destino (Izcara, 2013: 35). De no lograr lo anterior, el migrante retorna a su país y comunidad de origen.

La nueva economía de la migración es una continuidad de la teoría anterior, pero con otro enfoque (García, 2003: 343). La teoría considera que no es el individuo, sino la familia donde las personas actúan al momento de buscar la maximización y la diversificación del ingreso migrando a otro país porque en su país de origen no encuentran las condiciones para hacerlo; es por eso que entre más desigualdad exista en una comunidad, mayor será el incentivo para migrar (Arango, 2003: 12). Bajo esta teoría, el retorno se da cuando el migrante alcanzó sus objetivos económicos. Considerando el enfoque de la nueva economía de la migración laboral, Izcara (2011: 107) encontró que los migrantes rurales de Tamaulipas a Estados Unidos retornan porque la precariedad laboral a la que se enfrentan permite que su posición social sea más baja en el lugar de destino, pero más alta con respecto a sus paisanos en la comunidad de origen. 
De acuerdo con Mendoza (2013), una de las críticas que reciben la economía neoclásica y la nueva economía de la migración es que no abordan las condiciones sociales que influyen en la decisión de migrar y de retornar. Dando paso a nuevas teorías, desde la perspectiva sociológica, como la estructuralista, transnacionalista y la de redes sociales.

La teoría estructuralista considera la existencia de mercados laborales diferentes, por un lado, el centro que demanda mano de obra no calificada con salarios bajos que no se satisface en el mercado doméstico y por el otro lado la periferia que ofrece mano de obra abundante. Bajo esta teoría, la migración está delimitada por el intercambio desigual capitalista que se da entre el centro y la periferia (Castro-Escobar, 2016: 1569)

Pries (2002: 591) expone que la transmigración es una nueva forma de migración internacional que se caracteriza por una nueva relación entre espacios sociales y espacios geográficos; los transmigrantes no se identifican totalmente con su lugar de origen ni con el de destino ya que existen relaciones familiares o de amistad, por ejemplo, que permiten que el migrante se muevan de un país a otro, visto como un solo espacio social transnacional.

Por su parte, la teoría de las redes sociales considera que la decisión del retorno es afectada por los vínculos formales e informales en los países de origen, de tránsito y de destino del migrante (Orrego y Martínez, 2015: 36). Las redes se tejen entre familias, amigos y paisanos que se encuentran en diferentes países (García, 2003: 346), estas incrementan las posibilidades de migrar al disminuir los costos y riesgos del desplazamiento, además de incrementar los beneficios económicos (Massey et al., 1993: 448). Salas, Montoya y Soberón, (2011: 147) analizaron la migración de retorno en el Estado de México y documentaron la importancia de las redes sociales para la búsqueda de empleo en la primera migración de los mexiquenses.

Parte importante de las investigaciones realizadas sobre la migración de retorno han centrado su atención en el análisis del proceso de reintegración y/o reinserción, cuyo carácter puede ser tanto social como laboral. La reintegración ha sido abordada desde el carácter social, enfatizando el proceso donde el que ha retornado tiene que volver a integrarse a la sociedad a la cual se estaba anteriormente aculturado (Arowolo, 2000). 
Cuando se habla de la reinserción laboral, esta alude a un proceso posterior al retorno, donde los migrantes buscan dar continuidad a su trayectoria laboral como empleados o como empresarios en su lugar de origen (Anguiano-Téllez et al., 2013). El interés central de la presente investigación es analizar el carácter laboral de la reinserción, con base en la experiencia de estar de vuelta en su comunidad.

Diversos autores han tratado de identificar qué factores inciden en la reinserción laboral de migrantes retornados. Espinosa-Márquez y González-Ramírez (2016), por ejemplo, analizaron la reinserción social y laboral de los migrantes retornados de la comunidad de Atencingo, municipio de Chietla, Puebla, e identificaron cuatro grupos de retornados con características comunes: los dos primeros grupos con hasta 12 años de experiencia migratoria y con menos de 12 años de escolaridad, se desempeñaron principalmente como empleados del sector secundario y terciario; las personas con más de 15 años de migrante, de nueve a 12 años de escolaridad, emprendieron algún negocio; el grupo con 15 a 33 años de migrante y de 6 a 9 años de escolaridad, se emplearon en actividades del sector primario.

En la misma entidad, Corona-Jiménez (2018) analizó el retorno en Cuautlancingo, una comunidad urbana, muestra que 18 por ciento de los retornados emprendieron un negocio y el restante se emplearon fuera o dentro del municipio; antes, durante y después de migrar, los retornados se desempeñaban dentro del sector terciario, esto se explica porque se trata de un municipio con vocación industrial.

Solís (2018: 19), por su parte, analizó tres comunidades yucatecas (Tixbacab, Xohuayán y Buctzotz) y encontró una relación directa entre el tiempo de duración de la estancia en el extranjero y el ahorro e inversión; otro factor que influyó en el proceso de reinserción es la condición socioeconómica en la comunidad de origen: la vocación ganadera de Tixbacab permite que los migrantes inviertan en ganado, mientras que en Xohuayán solo ofrece oportunidades en la agricultura de temporal, por considerarse una zona maicera. Sin embargo, los migrantes de Tixbacab y Xohuayán que se emplearon en restaurantes de Estados Unidos, que aprendieron a preparar algunos platillos, no pueden reinsertarse laboralmente en sus comunidades porque no existen restaurantes donde puedan ofrecer sus servicios y emprender un negocio es difícil. El mismo caso lo enfrentan los retornados de Buctzotz, quienes aprendieron a construir con tablaroca, pero en su 
comunidad no pueden emplearse, lo que lo obliga a migrar a otros municipios como Mérida.

\section{BASES METODOLÓGICAS}

La presente investigación se realizó bajo el enfoque cualitativo, con alcance descriptivo y de corte transversal ya que se analizó para un periodo determinado. Dado que el objetivo fue describir el proceso de reinserción laboral de los migrantes retornados del municipio de Taniche, Oaxaca; el trabajo se llevó a cabo en tres etapas: 1) revisión de la literatura; 2) la investigación de campo utilizando como instrumento el cuestionario; ${ }^{2}$ y 3 ) procesamiento y análisis de resultados.

Para el diseño del instrumento de campo, se tomó como base los trabajos desarrollados por Garbey (2012), Ibarra (2016) y el Instituto de Estudios y Divulgación sobre Migración (2014), adaptándolos al contexto. Este se estructuró en siete secciones con 104 ítems con información cualitativa: perfil sociodemográfico, antecedentes laborales, historia migratoria, información sobre el último viaje que realizó el migrante a Estados Unidos, el retorno, la reinserción laboral y las expectativas de regreso a Estados Unidos.

Entre las dificultades más apremiantes presentadas en el trabajo de campo se encuentra la falta de información oficial que permitiera determinar con exactitud quiénes y cuántos migrantes retornados había en el municipio, por lo tanto, se optó por hacer un muestreo no probabilístico "bola de nieve". A partir de ello, se logró hacer contacto con 18 personas que retornaron en distintos momentos entre 1997 y 2017.

Para el análisis de los resultados se hace énfasis, principalmente, en la información proporcionada por los migrantes que retornaron en 2015-2017, ya sea de manera voluntaria o forzada, al ser este periodo donde las medidas antiinmigrantes se intensificaron en Estados Unidos, resultado de la batalla por la reforma migratoria como lo menciona Durand (2016), lo que dio como resultado un total de siete migrantes en el periodo determinado. En la presentación de los resultados, se aborda de manera general las características socioeconómicas del municipio y el fenómeno de migración; se caracteriza el perfil sociodemográfico de migrantes retornados para

2 Los nombres reales de las personas a quienes se les aplicó el cuestionario fueron cambiados para garantizar su confidencialidad. 
el periodo mencionado; y se narran procesos de reinserción laboral de los migrantes dentro del municipio.

\section{La comunidad de Taniche, Oaxaca: el contexto sociodemográfico}

Taniche, es uno de los 570 municipios de Oaxaca, pertenece al distrito Ejutla de Crespo y a la región Valles Centrales (ver Figura 1).

Figura 1. Localización del municipio de Taniche

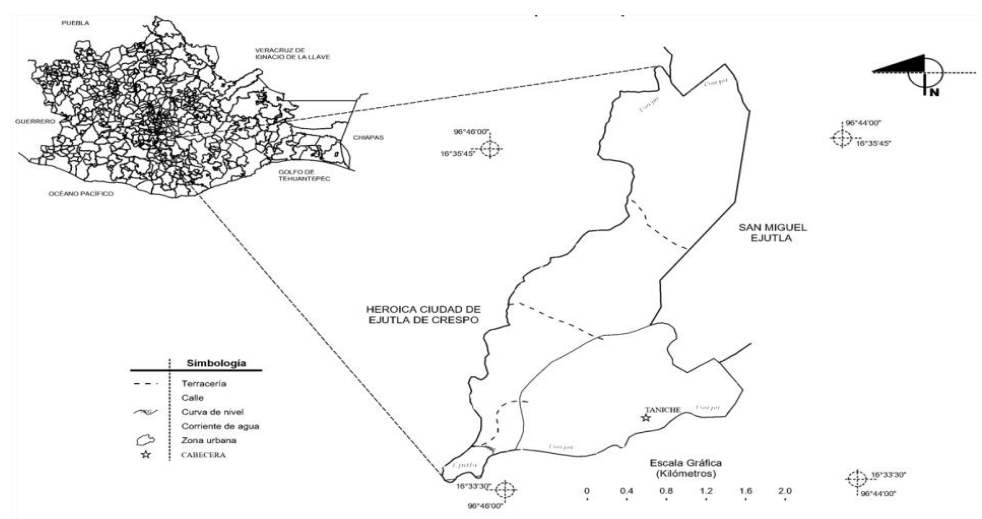

Fuente: Instituto Nacional de Estadística y Geografía (INEGI), 2005

De acuerdo con la Encuesta Intercensal realizada por el Instituto Nacional de Estadística y Geografía (2015), el municipio cuenta con una población de 825 habitantes, conformada mayoritariamente por mujeres (54.4 por ciento), distribuidas en 230 viviendas. De las 654 personas del municipio que se encuentran en edad de trabajar, 250 forman parte de la población económicamente activa, de las cuales 249 personas se encuentran ocupadas. Además, del total de personas ocupadas de Taniche 47.8 por ciento son trabajadores agropecuarios y 37 por ciento en actividades relacionadas con el comercio, ya sea como dueños o empleados de algún negocio.

La población del municipio se dedica preponderantemente a la siembra de maíz, frijol, calabaza, alfalfa, en menor medida el cultivo de caña, flores y hortalizas (jitomate, tomate y chile de agua); cabe resaltar que dichos cultivos son de temporal y riego, siendo este último de mayor número, ya que dentro del municipio se cuenta con pozos para riego y terrenos propicios para la agricultura. Otra actividad 
económica a la que se dedican, pero en menor medida, es la crianza y venta de animales de traspatio como gallinas, guajolotes, vacas, chivos, cerdos y/o borregos. Estas actividades se realizan en familia; desde el jefe de hogar, esposa e hijos, en muy pocas ocasiones se contratan ayudantes para la cosecha.

Las actividades agrícolas anteriormente descritas las hacen principalmente los hombres de la familia; quienes también desarrollan otras actividades a la par para obtener ingresos como jornalero, ayudante general o trabajador en la construcción. En el caso de las mujeres, en su mayoría se dedican a las labores del hogar (resultado de las pautas culturales de la división sexual del trabajo); y en algunas ocasiones compaginan su trabajo del hogar con empleos como empleadas domésticas o de algún micronegocio familiar (papelería, estética, panadería, abarrotes, café-internet, entre otros) para poder obtener ingresos.

Respecto a los ingresos, del total de la población ocupada, 51.4 por ciento gana hasta un salario mínimo, 21.3 por ciento de uno a dos salarios y solo 16.1 por ciento percibe más de dos salarios mínimos. Si comparamos los ingresos por sexo se identifica que el 72.9 por ciento de las mujeres perciben de uno a dos salarios mínimos, mientras que en el caso de los hombres el 69.6 por ciento percibe las misma cantidad por su trabajo (INEGI, 2015). La mayoría tiene empleos informales, por lo que no tiene prestaciones laborales ni un ingreso fijo para la manutención de su familia, no obstante, la dedicación a dichas ocupaciones es de tiempo completo.

De acuerdo con el Consejo Nacional de Población (CONAPO, 2015), el municipio presenta alto grado de marginación y un alto grado de rezago social (ver Tabla 1). Además, según datos del Consejo Nacional de Evaluación de la Política de Desarrollo Social (CONEVAL, 2015), 52.5 por ciento de la población del municipio se encuentra en pobreza moderada y 30.5 por ciento en pobreza extrema.

Respecto a los datos migratorios del municipio, este presenta un grado absoluto de intensidad migratoria alto; ocupa los lugares 91 de 570 en el contexto estatal y 321 de 2456 a nivel nacional (CONAPO, 2014). Muestra movimientos migratorios dentro y fuera del país, teniendo como destinos la capital oaxaqueña, la Ciudad de México y Estados Unidos (Texas y Atlanta, en su mayoría), principalmente como consecuencia de los bajos ingresos percibidos en la comunidad, en ese sentido, la migración se vuelve una vía para intentar una mejor calidad de vida. Con relación a la región donde se ubica la comunidad (Valles 
Centrales), se encuentra dentro del 9.92 por ciento de los municipios con intensidad migratoria alta (12 de 121 municipios); y considerando el distrito donde se ubica (Ejutla), es el único municipio que presenta esta característica ${ }^{3}$ (CONAPO, 2014).

Tabla 1. Características de población y vivienda, Taniche, Oaxaca, 2015

\begin{tabular}{lr}
\hline \multicolumn{1}{c}{ Características } & Porcentaje \\
\hline Población & \\
Con 15 años o más analfabeta & 11.9 \\
De 6 a 14 años que no asiste a la escuela & 7.6 \\
De 15 años o más con educación básica incompleta & 76.2 \\
Sin derechohabiencia a servicios de salud & 15.6 \\
Vivienda & \\
Con piso de tierra & 20.4 \\
No disponen de agua entubada de la red pública & 2.6 \\
Sin drenaje & 0.4 \\
Sin energía eléctrica & 30.0 \\
No disponen de lavadora & 1.7 \\
No disponen de refrigerador & 65.2 \\
\hline
\end{tabular}

Fuente: Elaboración propia con datos del CONEVAL (2015).

\section{Migrantes de TANiche: PERFIL SOCIODEMOgRáfico, EMIGRACIÓN Y RETORNO}

Los migrantes que retornaron a la comunidad de Taniche, en el periodo de 1997-2017, tuvieron como principales ciudades de destino, al momento de migrar, las siguientes: ciudad de Chino, condado de San Bernardino, en el estado de California (55 por ciento); Atlanta, Georgia (16.6 por ciento) y el restante el de otros estados como Texas y Pensilvania. Esto sugiere la actuación de las redes sociales en la definición del lugar de destino del migrante, más aún cuando se trata de una comunidad relativamente pequeña donde existe una relación de parentesco y amistad estrecha como Taniche porque, como señala Mora (2013: 30), las redes sociales ayudan en la definición del destino de los migrantes, también influye en la duración de la estancia en el

3 Es importante resaltar que este factor fue determinante en la selección del municipio, pero también su grado de marginación, así como la posibilidad de acceso a la información en campo dada su cercanía de los investigadores a dicho municipio. 
extranjero, posibles empleos y a extinguir inseguridades y miedos de los migrantes.

Las redes sociales de los migrantes se van tejiendo con amigos familiares y paisanos. Tal es el caso de J. García de 35 años de edad, quien ha migrado una sola ocasión a Estados Unidos. Antes de migrar trabajaba en el campo en compañía de su padre, pero en 2007 decidió emprender la salida de su comunidad, como resultado de las pláticas y apoyo que recibía por parte de su hermano que residía en Estados Unidos, decidió irse a Atlanta en donde trabajó durante ocho años en una fábrica (J. García, comunicación personal, 18 de diciembre de 2017).

De los siete migrantes que retornaron durante el periodo 20152017, se analizan características (ver Tabla 2) tales como: edad, escolaridad, estado civil y parentesco.

Tabla 2. Características sociodemográficas de los migrantes retornados

\begin{tabular}{lc}
\hline \multicolumn{1}{c}{ Características } & Frecuencia \\
\hline Parentesco con el jefe(a) del hogar & 2 \\
Jefe de hogar & 4 \\
Hijo(a) & 1 \\
Pareja & \\
Edad & 1 \\
20 - 30 & 2 \\
31 - 40 & 4 \\
41 - 50 & \\
Escolaridad & \\
Sin estudios & 1 \\
Primaria no terminada & 2 \\
Primaria & 1 \\
Secundaria no terminada & 2 \\
Media superior no terminada & 1 \\
Estado civil & \\
Casado(a) & \\
Soltero(a) & 4 \\
\hline
\end{tabular}

Fuente: Elaboración propia con datos del cuestionario aplicado en la comunidad. 
Como se observa en la tabla anterior, se trata de población adulta y en edad productiva, la mayoría se encuentra en los rangos 25-40 años (cuatro casos), seguidos por 41-51 años (tres casos); quienes iniciaron su proceso de migración a temprana edad como resultado de la falta de oportunidades educativas y laborales en su contexto. Se identificó, además, que la mayoría está casado(a). Respecto al parentesco con la jefatura del hogar, en su mayoría son jefes (as) del hogar (cuatro casos). El tamaño promedio de la familia es de cinco miembros, además de indicar que tienen familiares directos (padres, hijos o hermanos) que viven actualmente en Estados Unidos.

Un aspecto importante por mencionar es que su historia migratoria está formada por más de dos viajes y, en la mayoría de los casos, la estancia en el país vecino se fue alargando como resultado del incremento de los riegos de cruzar la frontera de manera indocumentada (ver Tabla 3).

Tabla 3. Características de la migración

\begin{tabular}{lc}
\hline \multicolumn{1}{c}{ Característica } & Frecuencia \\
\hline Duración de la última migración & \\
1- 4 años & 1 \\
$5-8$ & 3 \\
$9-12$ & 1 \\
$13-16$ & 0 \\
Númés & 2 \\
$1-2$ & 5 \\
3 - 4 & 1 \\
5 o más & 1 \\
\hline
\end{tabular}

Fuente: Elaboración propia con datos del cuestionario aplicado en la comunidad.

Uno de los retornados con mayor experiencia migratoria es J. Fernández quien mencionó que, a sus 42 años, ha cruzado siete veces la frontera entre México-Estados Unidos; la mayoría de las ocasiones lo efectuó como indocumentado, salvo la antepenúltima que fue de manera legal, por medio de un programa de trabajadores temporales (J. Fernández, comunicación personal, 18 de diciembre de 2017).

Otra de las personas que migró a edad temprana es F. González (comunicación personal, 18 de diciembre de 2017), casado y con tres hijos; estudió hasta la primaria, ha migrado dos veces a Estados Unidos. La primera vez que lo hizo tenía 15 años, estuvo cinco años trabajando en jardinería hasta que regresó al municipio; el empleo lo consiguió por medio de su hermano. En su segunda migración llegó al 
mismo lugar porque ahí ya tenía un trabajo. Regresó a su comunidad en 2015 por razones familiares (para cuidar a su esposa e hijos).

Las redes sociales influyeron en la búsqueda de empleo para D. Martínez. A sus 50 años está soltero y con estudios de nivel medio superior. Antes de migrar a Estados Unidos no trabajaba pues recientemente había terminado sus estudios; ha ido varias veces al país vecino, al principio iba cada dos años. La primera vez que viajó fue en 1992, llegó a Chino, California donde laboró en el cultivo de cebolla y en la jardinería, trabajos que consiguió por medio de unos amigos; trabajaba ocho horas, ganando cinco dólares por hora. La última vez que emigró a Estados Unidos fue en 2009, vivió en California por seis años, desempeñando el oficio de jardinero, donde trabajaba 45 horas semanales y ganaba 15 dólares por hora (D. Martínez, comunicación personal, 18 de diciembre de 2017). En 2016 regresó para estar con su familia.

Dentro de los motivos principales para emigrar (ver Tabla 4), se distingue la situación económica del hogar. Tal es el caso de una de las personas quien expresó: “allá pagan mejor, así que pueden hacer algo, porque aquí solo sale para comer..." (J. Fernández, comunicación personal, 18 de diciembre de 2017). Los migrantes buscan otras fuentes de ingreso, pues al no poder cubrir sus necesidades básicas del hogar, laboran en sus comunidades de origen (por la escasez de trabajo o bajos salarios), optan por migrar. Sin embargo, se identifica que la salida del lugar de origen también se da por la falta de oportunidades educativas, principalmente, seguida por razones en donde el sueldo no es suficiente para cubrir las necesidades básicas de la familia.

En relación con los motivos de regreso (ver Tabla 4), el retorno está relacionado en su mayoría por motivos familiares; en específico regresaron por enfermedad o muerte de un familiar o porque su esposa e hijos los necesitaban; una retornada indica,

nos avisaron que mi suegro había muerto, mi esposo me dijo que se regresaba a cuidar a su mamá, así que regresé al pueblo, junto con mi esposo y solo mis hijas más pequeñas (M. Hernández, comunicación personal, 18 de diciembre de 2017).

En el caso de M. Hernández, de 50 años de edad, estudió hasta la primaria y tiene cinco hijos. Llegaron a Atlanta, Georgia en donde consiguió dos trabajos (en una panadería y en una florería) donde aprendió a realizar arreglos florales, sin embargo, poco después se quedó con el trabajo de las flores naturales ya que consideraba que 
era muy cansado tener dos empleos, además de atender a su familia. En 2015, recibió la noticia de que su suegro estaba enfermo y al poco tiempo falleció, por lo que su esposo decidió regresar para apoyar en esos momentos a su mamá, es así como regresó a lado de su esposo e hijas pequeñas que nacieron en Estados Unidos y quedando sus hijas mayores en Atlanta (M. Hernández, comunicación personal, 18 de diciembre de 2017).

Tabla 4. Principales motivos de emigración y retorno

\begin{tabular}{lr}
\hline \multicolumn{1}{c}{ Característica } & Frecuencia \\
\hline Causa de la primera emigración & \\
Por deudas & 3 \\
Búsqueda de trabajo & 1 \\
Ir a conocer & 1 \\
Construir una casa & 1 \\
Situación económica & \\
Retorno involuntario & 1 \\
Deportación & \\
Retorno voluntario & 2 \\
Extrañar a esposa e hijos & 1 \\
Enfermedad/muerte de un familiar & 2 \\
Cuidar a sus padres adultos & 1 \\
Cumplimiento de meta & \\
\hline
\end{tabular}

Fuente: Elaboración propia con datos del cuestionario aplicado en la comunidad.

S. Rodríguez, por ejemplo, de 33 años de edad, estudió hasta la secundaria; ha ido dos veces a los Estados Unidos. La primera vez que salió fue en 2004, estuvo siete años fuera de su casa, llegó a Chino, California donde trabajó como soldador 10 horas al día, ganando 11 dólares por hora. La última vez que emigró fue en 2011, donde llegó a la ciudad de Rialto, condado de San Bernardino, donde vivió por seis años, pero debido a su trabajo se trasladaba a distintos lugares, es por eso que antes del retorno se encontraba en Pensilvania (S. Rodríguez, comunicación personal, 18 de diciembre de 2017). En 2017 decide regresar por la nostalgia de estar lejos de sus padres.

D. Martínez (comunicación personal, 18 de diciembre de 2017) mencionó que salió de su municipio para irse con unos familiares que vivían en Nezahualcóyotl, Estado de México, trabajaba en una escuela en donde tenía prestaciones y contrato laboral, pero decidió irse al país vecino donde vivió 16 años y regresó para cuidar a sus padres. 
Esto, coincide con datos de INEGI (2015: 31) donde se indica que la reunificación familiar es la principal causa del retorno de las personas a México. En el caso de los hombres, estos manifiestan que regresan ante la falta de trabajo (23.9 por ciento) y la deportación (9.2 por ciento), mientras que las mujeres regresan por la conclusión de los estudios (21.5 por ciento) y la falta de empleo (10.8 por ciento).

\section{REINSERCIÓN LABORAL DE LOS MIGRANTES RETORNADOS DE TANICHE}

En el análisis del proceso de reinserción laboral, se halló que cuatro personas se insertaron en labores agrícolas, dos como trabajadores del gobierno municipal y una en labores del hogar. El tiempo que pasó entre su regreso a la comunidad y lograr su reinserción laboral fue relativamente corto porque en su mayoría fue por cuenta propia dentro del sector agropecuario (ver Tabla 5).

Tabla 5. Características del trabajo actual de las personas retornadas

\begin{tabular}{lccc}
\hline \multicolumn{1}{c}{ Características } & Total & Características & Total \\
\hline Desde su regreso ha trabajado & & $\begin{array}{l}\text { Tiempo transcurrido entre su } \\
\text { regreso y que empezó a trabajar }\end{array}$ \\
Si & 6 & Inmediatamente & 5 \\
No & 1 & Un mes & 1 \\
¿Cómo consiguió su empleo en & & Dos meses & 1 \\
Taniche? & & ¿Cuántos empleos ha tenido & \\
Por cuenta propia & 6 & 1 & 5 \\
Amigos & 1 & 2 & - \\
& & 3 & 2 \\
\hline
\end{tabular}

Fuente: Elaboración propia con datos del cuestionario aplicado en la comunidad.

Respecto a las características del trabajo actual de los retornados, cinco de los siete casos de migrantes retornados, no tienen prestaciones laborales; dado que la mayoría trabaja por cuenta propia en el campo, de tiempo completo y no tienen un ingreso fijo. Solo en dos casos (trabajadores del ayuntamiento) tienen prestaciones laborales (vacaciones y dietas), trabajan medio tiempo y su salario es en promedio de mil 100 pesos semanales (ver Tabla 6).

Los migrante retornados, si bien logran insertarse rápidamente al ámbito laboral, terminan siendo absorbidos por el mismo contexto que los orilló a emigrar, pues regresan a una comunidad rural donde la 
oferta de trabajo es escasa, enfrentándose a condiciones de precariedad laboral. En el caso de Taniche, la mayoría de los retornados trabaja en actividades primarias, en específico en la siembra de granos (maíz y frijol) para su consumo y en menor medida para la venta. Estas condiciones han llevado a algunos autores (Márquez, Ordaz y Li, 2012; Peña, 2015) a cuestionar la existencia de una reinserción laboral.

Las condiciones en las que se reinsertan los migrantes, parece ser reflejo de lo que sucede a nivel nacional, tal como lo muestra un estudio realizado por Márquez, Ordaz y Li (2012: 260), quienes encontraron que entre 60 y 70 por ciento de los migrantes, que retornaron a México en el periodo 2005-2011, no tenían prestaciones laborales en su primer empleo; en el caso de los retornados rurales alrededor de 90 por ciento carecía de dichas prestaciones. Lo anterior se debe a que las personas que retornaron se emplearon en el sector informal.

Tabla 6. Características de su trabajo actual

\begin{tabular}{lclc}
\hline \multicolumn{1}{c}{ Característica } & Total & \multicolumn{1}{c}{ Característica } & Total \\
\hline Trabajo actual & & Prestaciones laborales & \\
Trabajador del ayuntamiento & 2 & Si & 2 \\
Campo & 4 & No & 5 \\
Ama de casa & 1 & & \\
Horas trabajadas por día & & Ingresos por trabajo (semanal) & \\
Tiempo completo & 4 & No tiene un ingreso fijo & 5 \\
1-5 horas & 1 & $1000-2000$ & 2 \\
6-10 horas & 1 & & \\
11-15 horas & 1 & & \\
\hline Fuente: Elaboración propia con datos del cuestionario aplicado en la comunidad.
\end{tabular}

Se encontró, además, que los retornados durante su estancia en Estados Unidos adquirieron habilidades entre las que destacan: el mantenimiento de las áreas verdes (jardinería y campo de golf), soldar o el cuidado de caballos en el caso de los hombres, mientras que la mujer aprendió a hacer arreglos florales (ver Tabla 7). Sin embargo, al analizar otro de los aspectos importantes dentro de la investigación relacionado con la posibilidad real de que estos utilicen conocimientos, habilidades y/o capital financiero adquirido durante la experiencia migratoria, se observa que a su regreso difícilmente pueden aprovechar lo aprendido porque el contexto en el que se reinsertan no demanda esas habilidades. 
Tabla 7: Habilidades adquiridas por los retornados

\begin{tabular}{lclc}
\hline Característica & Total & \multicolumn{1}{c}{ Característica } & Total \\
\hline $\begin{array}{l}\text { Considera que adquirió habili- } \\
\text { dades en Estados Unidos }\end{array}$ & & Habilidades que aprendió & \\
$\mathrm{Si}$ & 7 & Mantenimiento de campo de Golf & 1 \\
No & - & Soldar & 1 \\
& & Florería & 1 \\
& Jardinería & 2 \\
& & Fábrica (Empaquetar mercancía) & 1 \\
& & Cuidado de caballos & 1 \\
Aplica lo aprendido en Taniche & & Razones por lo que implementa lo \\
& & aprendido a su regreso & \\
Si & - & No es el mismo contexto & 3 \\
No & No en mi comunidad, pero sí en & 1 \\
& & otro lugar (México) & \\
& & Es diferente y más difícil & 3
\end{tabular}

Fuente: Elaboración propia con datos del cuestionario aplicado en la comunidad.

El señor J. Fernández, por ejemplo, desde que regresó a Taniche se incorporó a laborar en la comunidad, ha tenido tres empleos entre ellos ayudante de albañil y actualmente es trabajador del ayuntamiento, labor que desempeña de manera simultánea con las actividades agropecuarias por cuenta propia. Las habilidades adquiridas durante la migración en Estados Unidos no las ha podido aplicar. Allá aprendió a dar mantenimiento a los campos de golf y cosechar nuez; regresó a su comunidad porque sus padres estaban enfermos y lo necesitaba su esposa e hijos, además de indicar que extrañaba "su tierra" (J. Fernández, comunicación personal, 18 de diciembre de 2017).

Un caso similar al anterior es el que presenta M. López que a sus 49 años ha migrado dos veces a Estados Unidos, la primera vez llegó a Chino, California, en donde se empleó en la jardinería; trabajaba en promedio 10 horas diarias, ganando 400 dólares a la semana. La última vez que migró fue en el año 2000 , residió durante 16 años allá; se fue de manera indocumentada donde se estableció en Atlanta, Georgia, para trabajar en la jardinería. En 2017, las autoridades estadounidenses lo detienen y deportan, por lo que tuvo que regresar dejando a su familia; sin embargo, indicó que no tiene el interés de regresar "porque allá la ley es distinta", a su regreso está laborando en "el campo" (M. López, comunicación personal, 18 de diciembre de 2017)... El señor M. López mencionó que lo aprendido en el extranjero no lo puede implementar 
dentro de su municipio porque las condiciones del contexto son distintas.

En el caso de M. Hernández, quien trabajó en Estados Unidos en la panadería y en la florería, a su regreso se hizo cargo de su familia, además de administrar una tienda de abarrotes en su casa, al preguntarle por qué no realizaba lo que aprendió en la florería en Estados Unidos mencionó que en Taniche las personas no compran regularmente arreglos florales (M. Hernández, comunicación personal, 18 de diciembre de 2017). Al respecto, García y del Valle (2016: 10), mencionan que, en el caso de la reinserción laboral femenina, las mujeres se enfrentan a una dificultad adicional a la de los hombres porque el entorno familiar y comunitario las presiona para subordinarse a reproducir sus funciones tradicionales en el hogar.

Como se observa en las narraciones presentadas, el proceso de reinserción se realiza en un contexto de pobreza y marginación, por lo que los retornados tienen que volver a desarrollar las mismas actividades que tenían antes de migrar. Es importante considerar, como se indicó anteriormente, que la población de Taniche desarrolla actividades del sector primario, realizando labores propias del campo como es la siembra de maíz, frijol, calabaza y alfalfa; y en menor medida el cultivo de caña, verduras y flores que son vendidos en el distrito de Ejutla y para el autoconsumo. Aun cuando en muchos de los casos los migrantes se insertaron al sector primario, las condiciones en las que trabajaron distan mucho de las del contexto al que vuelven. Solo en un caso se logra apreciar la implementación de dichas habilidades, pero no dentro del contexto, sino que para ello debe de volver a migrar de manera interna.

Otro factor es que al no contar con capital económico esto les dificulta poder establecer algún emprendimiento donde apliquen lo aprendido, pues el regreso en muchos casos no es planeado, lo que les impide tener algún ahorro para invertir a su regreso. Es importante resaltar que la poca dinámica económica que existe en el territorio se basa en la oferta y demanda productos de consumo que satisfacen necesidades básicas, por lo que la demanda de productos como los arreglos florales o servicios de mantenimiento de jardines, que son algunas de las habilidades que adquirieron los migrantes retornados en Taniche, no son solicitados.

El escenario al que se enfrentan los migrantes en su regreso al municipio impide que puedan aprovechar su experiencia migratoria, 
aun cuando estos hayan adquirido nuevas habilidades y desarrollado nuevas capacidades durante su estancia en el extranjero, al volver no tienen las condiciones necesarias para que trabajar en actividades similares a lo aprendido y con ello implementar las capacidades.

\section{CONCLUSIONES}

Los resultados de la dinámica del retorno de los migrantes y su reinserción laboral en el municipio de Taniche, evidencian una cara de la realidad que se reproduce en muchas comunidades oaxaqueñas. Una vez analizado el proceso de reinserción se puede concluir que las condiciones laborales de los retornados son precarias y reproducen las mismas condiciones de las cuales huyen al momento de emigrar. En el caso de Taniche, los retornados continuaron laborando dentro del sector primario, consideradas como actividades informales, pues regresan a una comunidad rural donde la oferta de trabajo es escasa. Solo dos lograron una verdadera reinserción laboral al encontrar empleo en el ayuntamiento.

Los factores que intervienen en el proceso de reinserción laboral de migrantes retornados en el municipio de Taniche son principalmente: el tiempo de estancia en el extranjero, el motivo de regreso, el nivel educativo y el contexto local. En lo que se refiere al primer factor, la mayoría de los migrantes estuvieron por más de cinco años o hasta por más de tres lustros fuera de su comunidad. A pesar de tiempo que estuvieron fuera de su tierra a su regreso no cambiaron de empleo ni aplicaron lo aprendido en Estados Unidos. Respecto al segundo factor, la mayoría regresó por imprevistos familiares, por lo tanto, los migrantes no lograron prepararse para su regreso, es decir, no estaban listos para volver, pero las circunstancias los orillaron a regresar a su casa, por lo que no lograron revertir su situación a su regreso.

El tercer y cuarto factor se hace énfasis en la posibilidad real de que estos utilicen conocimientos, habilidades y capital financiero durante la experiencia migratoria. En el nivel educativo, se encontró que la mayoría de los retornados tienen educación básica, lo que provocó que durante su estancia en el país vecino desempeñaran trabajos pocos calificados; sin embargo, lo que aprendieron no es posible desarrollarlo en su comunidad.

Lo anterior, tiene amplia relación con el factor del contexto del municipio, pues todos los retornados declararon que no pueden implementar lo aprendido en el proceso migratorio, simplemente 
porque el contexto es distinto y que, aunque desempeñaron trabajos semejantes en Estados Unidos y Taniche, no se utilizan las mismas herramientas y las condiciones del lugar son distintas. Se observó que a su regreso difícilmente pueden aprovechar lo aprendido porque el contexto de pobreza y marginación en el que se reinsertan no demanda esas habilidades, dado que vuelven a una comunidad rural cuya oferta y demanda se enfoca solo en satisfacer necesidades básicas de consumo. Además, de que vuelven a un lugar con pocas ofertas laborales de tipo formal.

Con base en los resultados obtenidos, toma relevancia la actuación por parte de las autoridades en los distintos niveles, a través de la realización de programas o proyectos que contribuyan al aprovechamiento de las habilidades que adquirieron los retornados en el extranjero.

\section{REFERENCIAS BIBLIOGRÁFICAS}

Alarcón, R. (2012). "El debate sobre la migración cero". Observatorio de Migración Internacional, Letras migratorias Newsletter, CONAPO, 1-8.

Anguiano-Téllez, M., Cruz-Piñeiro, R. y Garbey-Burey, R. (2013). "Migración internacional de retorno: trayectorias y reinserción laboral de emigrantes veracruzanos". Papeles de población, 19 (77), 115-147.

Arango, J. (2003). "La explicación teórica de las migraciones: luz y sombras". Migración y desarrollo, (1), 1-30.

Arowolo, 0. (2000). "Return migration and the problem of reintegration". International migration, 38 (5), 59-82.

Barbosa, P. y Aguirre, J. (2011). "Migración de retorno e inversión en México". El caso de los migrantes de segunda generación. INCEPTUM, VI (10), 95-123.

Calva, L. y Alarcón, R. (2018). "Migrantes mexicanos deportados y sus planes para reingresar a Estados Unidos al inicio del gobierno de Donald Trump". Revista mexicana de ciencias políticas y sociales, 63 (233), 43-68.

Cassarino, Jean-Pierre (2004). "Theorizing Return Migration: The Conceptual Approach to Return Migrants". Revisited. International Journal on Multicultural Societies, 6 (2), 253-279.

Cassarino, Jean-Pierre (2008). "Entender los vínculos entre migración de retorno y desarrollo", en G. Pinyol (ed.), La dimensión exterior de las políticas de inmigración en la Unión Europea, CIDOB, Barcelona. 
Castillo, J. (1997). Teorías de la migración de retorno. En A. Izquierdo y G. Álvarez (Coord.), Políticas de retorno de emigrantes 29-44. España: Universidad de Coruña.

Castro-Escobar, E. (2016). Configuración de la migración interna en la región del Paisaje Cultural Cafetero de Colombia. Revista latinoamericana de ciencias sociales, niñez y juventud, 14(2), 1563-1585.

Cobo, S. (2008). ¿Cómo entender la movilidad ocupacional de los migrantes de retorno? Una propuesta de marco explicativo para el caso mexicano. Estudios Demográficos y Urbanos, 23(1), 159-177.

Consejo Nacional de Evaluación de la Política de Desarrollo Social. (CONEVAL). (2015). Medición de pobreza, anexo estadístico de pobreza a nivel municipio 2010 y 2015. Recuperado de https://www.coneval.org. $\mathrm{mx} /$ Medicion/Paginas/AE_pobreza_municipal.aspx

Consejo Nacional de Población. (2014). Índice absoluto de intensidad migratoria México Estados Unidos 2000-2010. Recuperado de https:// datos.gob.mx/busca/dataset/indice-absoluto-de-intensidad-migratoriamexico--estados-unidos-2000--2010

Consejo Nacional de Población. (2015). Datos abiertos del Índice de Marginación, por entidad federativa y municipios. Recuperado de http:// www.conapo.gob.mx/es/CONAPO/Datos_Abiertos_del_Indice_de_ Marginacion

Corona-Jiménez, M. (2018). El arduo proceso de reinserción laboral de los retornados en la periferia globalizada. Economía, sociedad y territorio, XVIII (57), 455-486.

d'Anglejan, S. (2009). Migraciones internacionales, crisis económica mundial y política migratoria. ¿Llegó la hora de retornar? OASIS, (14), 7-36.

Durand, J. (17 de julio de 2011). Migración a la baja. La jornada. Recuperado de https://www.jornada.com.mx/2011/07/17/opinion/019a2pol

Durand, J. (1994). Más allá de la línea. México: Consejo Nacional para la Cultura y las Artes.

Durand, J. (2004). Ensayo teórico sobre la migración de retorno. El principio del rendimiento decreciente. Cuadernos Geográficos, (35), 103116.

Durand, J. (2006). Los inmigrantes también emigran: la migración de retorno como corolario del proceso. REMHU - Revista Interdisciplinaria de Movilidad Humana, 14 (26 y 27), 167-189.

Durand, J. (2016). Historia mínima de la migración México-Estados Unidos. México: El Colegio de México. 
Espinosa-Márquez, A. y Gonzalez-Ramírez, M. (2016). "La adaptación social de los migrantes de retorno de la localidad de Atencingo, Puebla, México". CienciaUAT, 11(1), 49-64.

Gandini, L., Lozano-Ascencio, F. y Gaspar, S. (2015). El retorno en el nuevo escenario de la migración entre México y Estados Unidos. México: Consejo Nacional de Población y Secretaría de Gobernación.

Garbey, R. M. (2012). Retorno y reinserción laboral de emigrantes internacionales en Monte Blanco, Veracruz (Tesis de Maestría). El Colegio de la Frontera Norte, México.

García, R. (2003). "Un estado de la cuestión de las teorías de las migraciones". Historia contemporánea, (26), 329-351.

García, R. (2012). "Cero Migración: Declive de la migración internacional y el reto del empleo nacional" (Nota critica). Migraciones Internacionales, 6(4), 273-283.

García, R. y del Valle, R. (2016). "Migración de retorno y alternativas de reinserción. Hacia una política integral de desarrollo, migración y desarrollo humano". Huellas de la Migración, 1(1), 181-194.

Gómez, J. (2010). "La migración internacional: teorías y enfoques, una mirada actual". Semestre económico, 13(26), 81-99.

Ibarra, J. I. (2016). Inserción laboral y trayectorias de los migrantes mexicanos deportados en Tijuana (Tesis de Maestría). El Colegio de la Frontera Norte, México.

Instituto de Estudios y Divulgación sobre Migración. (2014). Proyecto piloto de México migración y empleo: reinserción de los migrantes de retorno al mercado laboral nacional. Consultado en http://www. estudiosdemigracion.org/wp-ontent/uploads/2017/03/2015_ Migracion_y_empleo.pdf

Instituto Nacional de Estadística y Geografía (INEGI). (2015). Encuesta Intercensal 2015. Consultado en https://www.inegi.org.mx/programas/ intercensal/2015/default.html\#Tabulados

Izcara, S. (2011). "La migración de retorno: los jornaleros tamaulipecos". Migración y desarrollo, 9 (17), 91-112.

Izcara, S. (2013). "Aproximación teórica al estudio de los procesos migratorios permanentes". Estudios sociales: Revista de Alimentación Contemporánea y Desarrollo Regional, 21(42), 27-54.

Jacobo, M. y Cárdenas, N. (2018). Los retornados: ¿Cómo responder a la diversidad de migrantes mexicanos que regresaron de Estados Unidos? México: Centro de investigación y docencia económicas.

Jáuregui, J. y Recaño, J. (2014). "Una aproximación de las definiciones, tipologías y marcos teóricos de la migración de retorno". Revista bibliográfica de geografía y ciencias sociales, XIX (1084), 1-32. 
Lozano, F. y Martínez, J. (2015). Retorno en los procesos migratorios de América Latina: conceptos, debates, evidencias. Río de Janeiro, Brasil: Asociación Latinoamericana de Población (ALAP).

Márquez, A., Ordaz, J. y Li, J. (2012). "Inserción laboral y características de los migrantes mexicanos de retorno 2005-2011". Comparación urbanarural. En T. Ramírez y M. Castillo (Coord.) El estado de la migración México ante los recientes desafíos de la migración internacional, 237-267. México: CONAPO.

Masferrer, C., Sánchez, L. y Rodríguez, M. (2017). "Condiciones laborales de los migrantes de retorno de Estados Unidos". Apuntes para la equidad. Colegio de México. Seminario sobre trabajo y desigualdad, (2), 1-4.

Massey, D., Arango, J., Graeme, H., Kouaouci, A., Pellegrino, A. y Taylor, E. (1993). "Theories of international migration: a review and appraisal". Population and development review, 19(3), 431-466.

Mendoza, J. (2013). "Migración de retorno, niveles educativos y desarrollo socioeconómico regional de México". Estudios Sociales, 21(42), 57-85.

Mora, D. (2013). "Metodología para la investigación de las migraciones". Integra educativa, VI(1), 13-42.

Organización de las Naciones Unidas para la Educación, la Ciencia y la Cultura. (2008). People on the move: Handbook of selected terms and concepts. Paris, Francia: Section on International Migration and Multicultural Policies. Consultado en https://unesdoc.unesco.org/ ark:/48223/pf0000163621

Orrego, C. y Martínez, J. (2015). "Retorno en la migración: una mirada a sus múltiples facetas". En F. Lozano y J. Martínez (Ed.), Retorno en los procesos migratorios de América Latina. Conceptos, debates, evidencias, 25-54. Brasil: Asociación Latinoamericana de Población.

Peña, J. (2015). "Perfil laboral de migrantes mexicanos deportados e inserción laboral en México". Migración y desarrollo, 13(24), 167-184.

Pries, L. (2002). "La migración transnacional y la perforación de los contendores de Estados-nación". Estudios demográficos y urbanos, (51), 571-597.

Rivera, L. (2008). "El eslabón urbano en el trayecto interno del circuito migratorio Mixteca-Nueva York-Mixteca: Los migrantes de Nezahualcóyotl, Estado de México". En E. Lavine (Ed), La migración y los latinos en Estados Unidos. Visiones y Conexiones, 53-73. México: Universidad Nacional Autónoma de México.

Rivera, L. (2011). “¿Quiénes son los retornados? Apuntes sobre el Migrante retornado en México contemporáneo". En B. Feldman-Bianco, L. Rivera, C. Stefoni y M. I. Villa (comps.), La construcción social del migrante en América Latina: Prácticas, representaciones y categorías, 309-338. Quito, 
Ecuador: CLACSO, Facultad Latinoamericana de Ciencias Sociales-sede en Ecuador, FLACSO y Universidad Alberto Hurtado.

Rivera, L. (2015). Narrativas de retorno y movilidad. Entre prácticas de involucramiento y espacialidades múltiples en la ciudad. Estudios Políticos, (47), 243-264.

Salas, R., Montoya, J.y Soberón, J. A. (2011). La migración internacional de retorno y su influencia sobre las formas de vida den el estado de México. CIMEXUS, 6(1), 135-156.

Solís, M. (2018). Aproximaciones al análisis de la precariedad laboral de la migración de retorno. Un estudio comparativo entre migrantes yucatecos. Norteamérica, 13(1), 7-32.

Unidad de Política Migratoria de la Secretaría de Gobernación de México (2018). Series históricas, Eventos de repatriación de mexicanos desde Estados Unidos, según entidad federativa y punto de recepción. Recuperado de http://www.politicamigratoria.gob.mx/es_mx/SEGOB/ Repatriacion_de_mexicanos_2017

Vásquez, S. (2016). Reinserción laboral de los migrantes de retorno en Yecuatla, Veracruz. Revista de Ciencias Sociales, (5), 201-219.

Zenteno, R. (2012). Saldo migratorio nulo: el retorno y la política antiinmigrante. Coyuntura Demográfica, (2), 17-21.

\section{RESUMEN CURRICULAR DE LOS AUTORES}

Teresa Griselda Lustre Díaz

Nacionalidad mexicana. Cursó la Licenciatura en Administración en la Universidad NovaUniversitas, actualmente se desempeña como técnico en la Universidad Nova Universitas ubicada en Ocotlán de Morelos, Oaxaca.

Dirección electrónica: lustre@live.com.mx

\section{Marco Antonio Espinosa Trujillo}

Nacionalidad mexicana. Cursó el doctorado en Economía en el Colegio de Postgraduados, actualmente se desempeña como profesorinvestigador en la Universidad NovaUniversitas ubicada en Ocotlán de Morelos, Oaxaca.

Dirección electrónica: marco_trujillo@outlook.com 
Elizabeth Ojeda Velasco

Nacionalidad mexicana. Profesora investigadora de tiempo completo en NovaUniversitas, campus central Ocotlán, Oaxaca, adscrita al departamento de Administración. Maestra en Economía Aplicada por el Colegio de la Frontera Norte (COLEF).

Dirección electrónica: elyizabeth.ov@gmail.com 